\title{
Úlcera penetrante de aorta ascendente en un paciente asintomático
}

\section{Penetrating ascending aortic ulcer in an asymptomatic patient}

\author{
A. Bayona ${ }^{1}$, E. Vallejo' ${ }^{1}$, R. Hernández-Estefanía ${ }^{2}$, G. Bastarrika ${ }^{3}$, G. Rábago ${ }^{2}$
}

\section{RESUMEN}

La úlcera penetrante de aorta (UPA) es la ulceración de una placa aterosclerótica que afecta a la lámina elástica interna de la aorta, y que puede evolucionar hacia un hematoma de pared o una disección aórtica si se produce el paso de sangre hacia la capa media. A pesar de que se localiza más frecuentemente en la aorta descendente, puede presentar una alta mortalidad en caso de situarse en la aorta ascendente, donde la cirugía está indicada aunque el paciente se encuentre asintomático. Presentamos el caso de un paciente sin sintomatología con úlcera penetrante de aorta ascendente (UPAA) ascendente sometido a sustitución de aorta ascendente por una prótesis vascular.

Palabras clave. Úlcera penetrante. Aorta ascendente. Raíz de aorta.

\begin{abstract}
Penetrating aortic ulcer (PAU) has been defined as an atherosclerotic plaque ulceration that breaks the internal elastic lamina of the aorta, which may progress to a wall hematoma or aortic dissection in case of blood seeping into the middle layer. Although PAU is commonly located in the descending aorta, the involvement of the ascending aorta can be fatal. Therefore, surgery is indicated even in asymptomatic patients presenting an ascending PAU. We report on an asymptomatic patient with ascending PAU referred for replacement of the ascending aorta with a composite prosthetic graft.
\end{abstract}

Keywords. Penetrating ulcer. Ascending aorta. Aortic root.
1. Facultad de Medicina. Universidad de Navarra. Pamplona.

2. Servicio de Cirugía cardiaca. Clínica Universidad de Navarra. Pamplona.

3. Servicio de Radiología. Clínica Universidad de Navarra. Pamplona.

\section{Correspondencia:}

Rafael Hernández Estefanía

Servicio de Cirugía cardiaca

Clínica Universidad de Navarra

Avda. Pío XII, 36

E-mail: r.hernandez.estefania@gmail.com

Recepción: 28 de febrero de 2012

Aceptación provisional: 6 de marzo de 2012

Aceptación definitiva: 6 de marzo de 2012 


\section{INTRODUCCIÓN}

La úlcera penetrante de aorta (UPA) es la ulceración de una placa aterosclerótica que afecta a la lámina elástica interna, y que puede evolucionar hacia un hematoma de pared o una disección aórtica si se produce el paso de sangre hacia la capa me$\mathrm{dia}^{1}$. La UPA es una entidad que forma parte del denominado Síndrome Aórtico Agudo (SAA), junto con la disección aórtica, el hematoma intramural y la rotura traumática de aorta ${ }^{2}$, que presentan las mismas manifestaciones clínicas, pero diferente etiopatogenia y tratamiento.

La UPA es la entidad menos prevalente entre los SAA y se localiza más frecuentemente en la aorta descendente ${ }^{3}$. A pesar de ser una entidad grave, con una alta mortalidad en fase aguda ${ }^{2}$, no existe un consenso en relación al tratamiento y/o métodos diagnósticos más adecuados para su manejo. De este modo, algunos autores preconizan el tratamiento quirúrgico urgente ${ }^{4}$, otros grupos consideran realizar procedimientos endovasculares inmediatos cuando la úlcera afecta a la aorta descendente $\mathrm{y}$, en determinadas ocasiones, se ha defendido un tratamiento conservador ${ }^{5}$. No existen tampoco criterios definidos con relación a la prueba de imagen más útil para el establecimiento del diagnóstico definitivo.

Se presenta el caso clínico de un paciente asintomático con úlcera penetrante de aorta ascendente (UPAA) sometido a sustitución de aorta ascendente por una prótesis vascular.

\section{CASO CLÍNICO}

Varón de 68 años de edad, exfumador, hipertenso, dislipémico y con antecedente de neoplasia de colon intervenida hace 5 años. En una revisión rutinaria se realiza un escáner de tomografía computarizada (TC) toraco-abdominal, donde se observa un engrosamiento concéntrico de la pared aórtica ascendente con varias úlceras, la mayor de $1,8 \times 0,9 \mathrm{~cm}$, localizada en la cara lateral izquierda de la aorta ascendente. De la misma manera, se observa junto a la úlcera un hematoma mural de 4,8 cm de longitud (Figs. 1 y 2). Aunque el paciente no refiere sintomatología, las imágenes son compatibles con UPAA por lo que se indica cirugía de sustitución de la aorta ascendente.
Bajo anestesia general, se realiza esternotomía media. Mediante implantación de dos cánulas -arterial y venosa- en arteria femoral y orejuela derecha, respectivamente, se establece circulación extracorpórea. Se pinza la aorta, se para el corazón con una dosis de cardioplejia anterógrada y se realiza aortotomía longitudinal. Una vez abierta, se observa una úlcera en la cara posteroizquierda de la aorta ascendente, a un centímetro del tronco arterial braquiocefálico, así como una disección aórtica circunferencial en el segmento de aorta correspondiente a la localización de la úlcera. Se decide realizar hipotermia profunda $\left(17^{\circ} \mathrm{C}\right)$ y paro circulatorio para resecar el segmento de aorta que contiene la rotura y que se prolonga hasta el tronco braquiocefálico (Fig. 3). Durante la parada circulatoria de 21 minutos, se realiza perfusión cerebral retrógrada mediante una cánula colocada en la vena cava superior. Se reemplaza la porción ascendente de la aorta por una prótesis vascular de Dacron de $3,4 \mathrm{~cm}$.

El postoperatorio transcurre sin incidencias y el paciente es dado de alta al octavo día. Diez meses después de la operación, el paciente realiza vida normal y no ha presentado complicaciones.

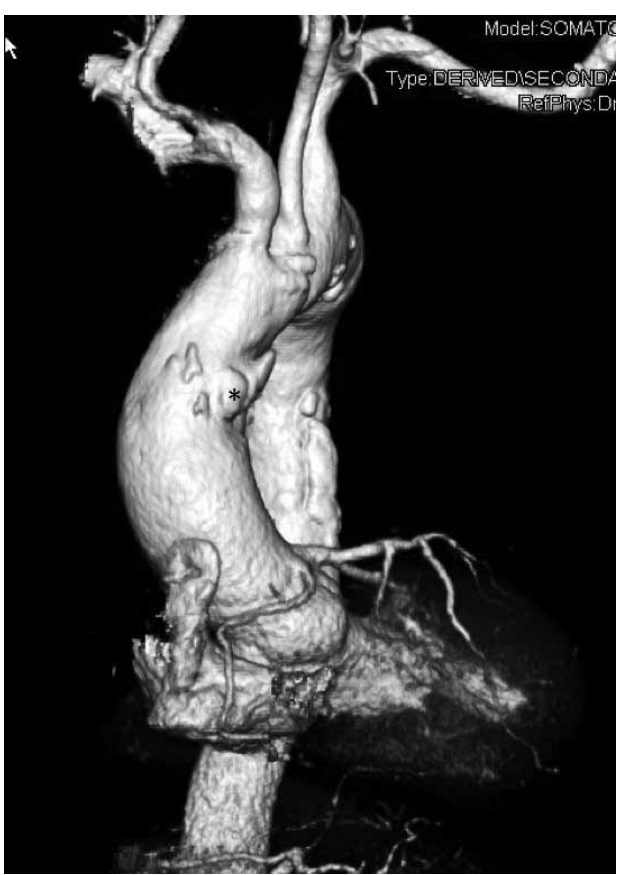

Figura 1. Reconstrucción mediante angioTAC de aorta ascendente, cayado y aorta descendente. La UPAA aparece como una protusión en la pared aórtica $\left({ }^{*}\right)$. 


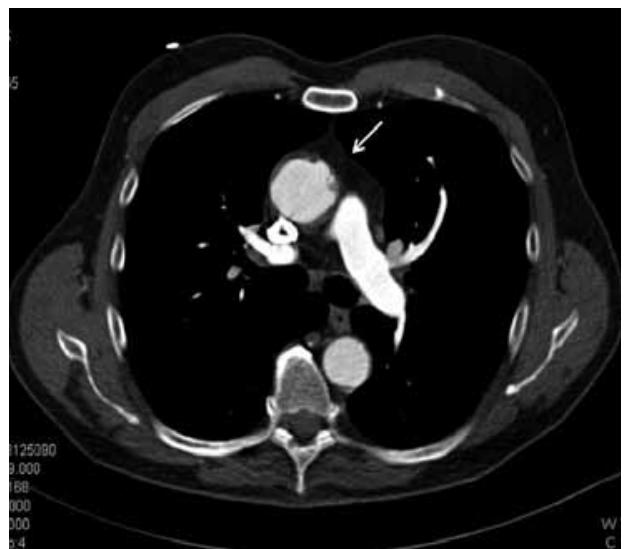

Figura 2. TAC con contraste a nivel de la aorta ascendente. Ulceración con salida de contraste hacia la pared aórtica (flecha).

\section{DISCUSIÓN}

La UPAA es una entidad que parece afectar por igual a hombres y mujeres a partir de la séptima década de la vida ${ }^{5-10,13}$, aunque a veces puede producirse en edades menos avanzadas ${ }^{4,11,12,14}$. Generalmente se asocia a hipertensión arterial (HTA) y enfermedad aterosclerótica avanzada ${ }^{5-12,14}$, aunque también aparece en pacientes con sífilis ${ }^{11}$, como complicación tardía de cirugía cardíaca ${ }^{5}$ o en enfermedades del tejido conectivo (como el síndrome de Marfan, o la enfermedad Ehlers-Danlos), donde está descrito que estas conectivopatías favorecen la dilatación de la aorta ascendente y la formación de UPAA ${ }^{4,15}$.

La UPA se localiza con mayor frecuencia en la porción media y distal de la aorta torácica descendente (90\%). Algunos autores lo atribuyen al efecto protector que proporciona el mayor flujo existente en la porción proximal de la aorta, evitando la formación de placas de ateroma ${ }^{16}$. En aorta ascendente, la UPA es menos frecuente (10\%), presentando incluso un peor pronóstico que la disección de aorta ${ }^{4,17}$. En relación a su situación en el eje anteroposterior, las úlceras de aorta ascendente pueden presentar una localización diversa, pudiendo ser anteriores ${ }^{4,10,14}$ o posteriores ${ }^{8,9}$.

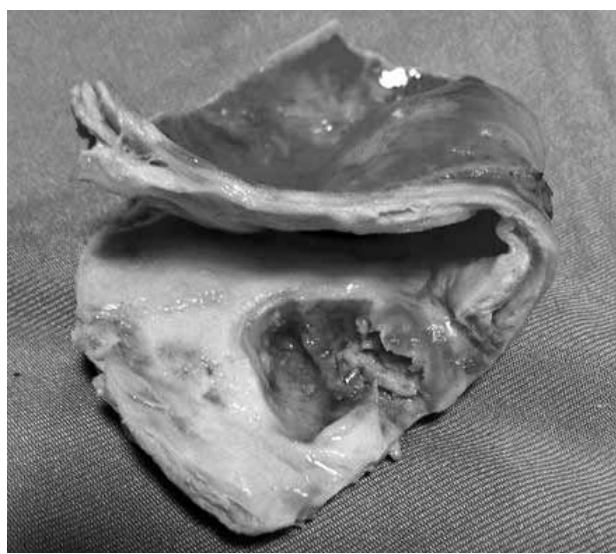

Figura 3. Fragmento de aorta ascendente donde se observa la ulceración del endotelio que afecta a la lámina elástica interna.

En el momento del diagnóstico, la UPAA puede encontrarse en diferentes fases de evolución (disección ${ }^{3}$ o hematoma'), dependiendo del grado de afectación de la pared de la aorta. Por este motivo, todos estos procesos se engloban en la actualidad en el denominado $\mathrm{SAA}^{2}$. El diagnóstico definitivo de UPAA sólo se obtiene mediante el análisis histológico de la pieza después de la cirugía ${ }^{7,8,14}$.

Los pacientes con UPAA suelen estar asintomáticos a menos que la lesión esté evolucionada y aparezcan complicaciones $^{6,8,12-14}$, que hagan necesaria cirugía urgente o pericardiocentesis por taponamiento cardíaco ${ }^{12}$. Cuando existen síntomas, el más frecuente es el dolor torácico, que puede confundirse con otras patologías, lo que hace necesario un análisis de sus características para el diagnóstico diferencial. La mayoría de pacientes con úlcera de aorta y síntomas, describen un dolor severo y punzante, similar al dolor lacerante que se suele asociar a otros SAA, como la disección de aorta, y que puede acompañarse de alteraciones de repolarización en el electrocardiograma (ECG) ${ }^{6,12,14}$.

A diferencia de la disección de la aorta, los pacientes con UPAA mantienen pulsos periféricos, aunque esta situación puede modificarse si la ulceración ha pro- 
gresado a disección o hematoma intramural $^{5,6}$.

Las pruebas de imagen resultan fundamentales debido a la dificultad en el establecimiento del diagnóstico a través de la clínica. En casi todos los pacientes se emplean varias técnicas ${ }^{5-10,12,14}$, siendo la ecografía transesofágica la más utilizada, y la TC con contraste la prueba que más frecuentemente proporciona el diagnóstico.

La angiografía parece actualmente relegada a un segundo plano ${ }^{12}$ debido al riesgo de rotura del endotelio en una aorta frágil, y tanto la radiografía como el ECG pueden mostrar alteraciones, pero ambas carecen de una alta especificidad ${ }^{8}$.

En general, la indicación quirúrgica en las enfermedades agudas de la aorta está condicionada por la localización de la úlcera, hematoma o disección ${ }^{1}$. De este modo, está bien establecido que las disecciones localizadas en la aorta ascendente tienen indicación quirúrgica emergente por el riesgo de rotura ${ }^{14,16}$. Existe mayor controversia en relación con el tratamiento de la UPA, y se ha sugerido que la distinción entre úlcera ascendente y descendente también es necesaria para determinar el procedimiento terapéutico más adecuado ${ }^{1}$. Si la UPA es ascendente, se recomienda cirugía de sustitución de la porción afectada de la aorta, mientras que si es descendente, el abordaje puede ser endovascular, siempre que no se presenten síntomas de progreso. La inestabilidad hemodinámica, persistencia o recurrencia del dolor, ruptura aórtica, embolismo distal, taponamiento cardíaco y aumento rápido del diámetro aórtico, entre otras $^{16}$, indicarían un abordaje quirúrgico, independientemente de la localización. El abordaje endovascular en la aorta ascendente puede ser un procedimiento demasiado arriesgado ${ }^{8}$.

A pesar del riesgo inherente a la intervención, la cirugía de la aorta ascendente presenta buenos resultados ${ }^{4,6-8,10,13}$, siendo probablemente el tratamiento más eficaz de la UPAA, aunque el paciente se encuentre asintomático. El tratamiento conservador no parece recomendable debido al potencial riesgo de complicaciones que puedan resultar fatales ${ }^{9}$.
En conclusión, la UPAA es una entidad poco frecuente que se asocia principalmente a enfermedad aterosclerótica, HTA, y dilatación de aorta ascendente. Puede cursar sin sintomatología o con dolor precordial y alteraciones electrocardiográficas, por lo que debe ser considerada en el diagnóstico diferencial del dolor torácico. El riesgo potencial de que la UPAA evolucione a rotura de aorta aconseja cirugía urgente, aunque el paciente se encuentre asintomático.

\section{BIBLIOGRAFÍA}

1. SundT TM. Intramural hematoma and penetrating atherosclerotic ulcer of the aorta. Ann Thorac Surg 2007; 83: 835-841.

2. Evangelista Masip A. Progress in the acute aortic syndrome. Rev Esp Cardiol 2007; 60: 428439.

3. Ganaha F, Miller DC, Sugimoto K, Do YS, MinaMiguchi H, SAITo H, et al. Prognosis of aortic intramural hematoma with and without penetrating atherosclerotic ulcer: A clinical and radiological analysis. Circulation 2002; 106: 342-348.

4. Hammoudi N, Dorfmuller P, Corvol E, Acar C. Penetrating ulcer of the ascending aorta without rupture. Eur J Cardiothor Surg 2009; 35: 550-551.

5. Jain D, Gehl HB, Katus HA, Richardt G. Penetrating atherosclerotic ulcer of the ascending aorta: A delayed complication of coronary artery bypass surgery a case report. Angiology 2003; 54: 251-255.

6. Bilge M, Kurt M, Senkaya EB, Yasar AS, Karakas F, Erayman A. Penetrating atherosclerotic aortic ulcer mimicking acute inferior myocardial infarction. Echocardiography 2009; 26: 10841086.

7. Casolo G, Lera J, Magnacca M, Chioccioli M, Tessa C. Aortic penetrating ulcers associated with intramural haematoma: Detection and evaluation by multislice computed tomography. Eur Heart J 2009; 30: 527.

8. Singhal P, Lin Z. Penetrating atheromatous ulcer of ascending aorta: A case report and review of literature. Heart Lung Circ 2008; 17 : 380-382.

9. Kimura S, Noda M, Usui M, Isobe M. Diagnostic criteria for penetrating atheromatous ulcer of the throracic aorta. Ann Thorac Surg 2004; 78: 1070-1072. 
10. BELKin RN, Kalapatapu SK, Lafaro RJ, Ramaswamy G, McClung JA, Cohen MB. Atherosclerotic pseudoaneurysm of the ascending aorta. J Am Soc Echocardiogr 2003; 16: 367-369.

11. Saleem MA, McNeeley M, Zias E, Pucillo A, Ro $\mathrm{JH}$, WeISS MB. Penetrating ulcer of the ascending thoracic aorta in syphilis. Catheter Cardiovasc Interv 2004; 61: 16-19.

12. Yano K, Makino N, Hirayama H, Hatakenaka M, Matsui H, Soeda T et al. Penetrating atherosclerotic ulcer at the proximal aorta complicated with cardiac tamponade and aortic valve regurgitation. Jpn Circ J 1999; 63: 228230.

13. Stamou SC, Kouchoukos NT. Penetrating ulcer of the ascending aorta. J Am Coll Cardiol $2011 ; 57: 1327$.
14. De Souza DG, Blank RS, Mazzeo FJ, Singh KE. Penetrating ascending aortic atherosclerotic ulcer. Anesth Analg 2009; 109: 1035-1037.

15. BAIKoussis NG, Apostolakis EE. Penetrating atherosclerotic ulcer of the thoracic aorta: Diagnosis and treatment. Hellenic J Cardiol 2010; 51: 153-157.

16. Castañer E, Andreu M, Gallardo X, Mata JM, Cabezuelo MA, Pallardó Y. CT in nontraumatic acute thoracic aortic disease: typical and atypical features and complications. Radiographics 2003; 23 Spec nº: S93-110.

17. Coady MA, Rizzo JA, Hammoudi GL, Pierce JG, Kopf GS, Elefteriades JA. Penetrating ulcer of the thoracic aorta: What is it? How do we recognize it? How do we manage it? J Vasc Surg 1998; 27: 1006-1016. 
